\title{
Psychoacoustical Estimation of Selected Acoustic Signals Which Can Be Applied at Pedestrian Crosswalks
}

\author{
E. Bogusz And A. Furmann \\ Institute of Acoustics, Adam Mickiewicz University, Umultowska 85, 61-614 Poznań, Poland
}

\begin{abstract}
The paper presents a psychoacoustical evaluation of selected acoustic signals, which according to Polish and international recommendations can be applied at pedestrian crosswalks. The aim of the study was the optimal adjustment of parameters of the signals emitted at pedestrian crosswalks, and to meet the expectations of persons with vision impairments as well as nearby residents or workers working near the crossing. The psychoacoustical evaluation of acoustic signals consists of determination of the hearing threshold of signals in the quiet, detection of signals presented against a background of various types of traffic noises (estimated signal-to-noise ratio) and annoyance assessment of signals. Three types of intermittent sounds were analysed: signals with rectangular time pattern filled up with rectangular wave, signals with rectangular time pattern filled up with sinusoidal wave and signals with triangular time pattern filled up with sinusoidal wave. Four fundamental frequencies $(550 \mathrm{~Hz}, 880 \mathrm{~Hz}, 1580 \mathrm{~Hz}$ and $2000 \mathrm{~Hz})$ and two repetition rates $(5 \mathrm{~Hz}$ and $9 \mathrm{~Hz})$ were tested. The results of the study have shown that the most suitable signals which can be applied at pedestrian crosswalks are the intermittent sounds with rectangular time pattern filled up with rectangular wave of fundamental frequencies $880 \mathrm{~Hz}$ and $1580 \mathrm{~Hz}$ and repetition rate $5 \mathrm{~Hz}$ and the intermittent sounds with rectangular time pattern and triangular time pattern both filled up with sinusoidal wave of fundamental frequencies $1580 \mathrm{~Hz}$ and repetition rate $5 \mathrm{~Hz}$.
\end{abstract}

PACS: 43.66.Cb, 43.66.Dc, 43.66.Ki, 43.66.Lj

\section{Introduction}

A lot of studies have shown a significant improvement in safety of vision impaired persons at the pedestrian crosswalks after introduction of audible pedestrian traffic signals $[1,2]$. The study carried out in California in 1991 [1] has shown that after installation of acoustic signalling devices generating audible traffic signals at the pedestrian crosswalks, the number of accidents involving vision impaired persons was reduced.

Undoubtedly, acoustic signalizations at pedestrian crosswalks are very helpful but on the other hand the systems of sound signalization applied today have many drawbacks. There is too large variety of signals informing about the time when crossing the street is allowed. The same acoustic signal at one pedestrian crosswalk can inform about a possibility to walk, while at another about the necessity to wait.

Review of both Polish and international literature [3-6] as well as many studies of acoustic signalization at the pedestrian crosswalks not published yet, has shown a large variety of signals recommended for the purpose. As the majority of the blind would prefer having a single signal, the choice of the most suitable signal which should be applied at pedestrian crosswalks is still an open problem.

The aim of the study was to establish the optimum parameters of acoustic signals to be emitted at pedestrian crosswalks. The selection should be made taking into account the expectations of persons with vision impair- ments (audible enough, readily perceived, unambiguous, one definite signal at all pedestrian crosswalks) as well as persons who live and work near pedestrian crosswalks (not annoying signal). The best choice should consider the type of a signal, its fundamental frequency and repetition rate, so that the signal is the best audible both in the quiet as well as against a background of traffic noise and it is not annoying.

Polish $[7,8]$ as well as international [9] normative regulations define a wide range of acoustic signals parameters that can be emitted at pedestrian crosswalks. The Polish directives recommend fundamental frequency from the range of $550-2000 \mathrm{~Hz}$, the repetition rate of the intermittent sound from the range of $5-12.5 \mathrm{~Hz}$ and the sound pressure level of signals between $50 \mathrm{~dB}$ and $85 \mathrm{~dB}$ (A). International normative regulations recommend signals which have high and low frequencies, from the range 300-3500 Hz. The repetition rate of the intermittent sound should be above $2.5 \mathrm{~Hz}$. The sound pressure level of signals should be $5-10 \mathrm{~dB}$ above traffic noise level. For this reason the aim of the study was to precisely describe parameters of a signal which we propose to use at pedestrian crosswalks. The parameters of the signals investigated in this study are in the range recommended by Polish and international regulations.

In the most popular installation of acoustic signalling devices at pedestrian crosswalks in Poznań a fundamental frequency could be chosen from sixteen frequencies: $100 \mathrm{~Hz}, 150 \mathrm{~Hz}, 230 \mathrm{~Hz}, 360 \mathrm{~Hz}, 550 \mathrm{~Hz}, 620 \mathrm{~Hz}, 700 \mathrm{~Hz}$, 
$780 \mathrm{~Hz}, 880 \mathrm{~Hz}, 990 \mathrm{~Hz}, 1110 \mathrm{~Hz}, 1250 \mathrm{~Hz}, 1410 \mathrm{~Hz}$, $1580 \mathrm{~Hz}, 1780 \mathrm{~Hz}$ and $2000 \mathrm{~Hz}$. There are also thirteen potential repetition rates: twelve from the range of $1 \mathrm{~Hz}$ to $12 \mathrm{~Hz}$ with a step of $1 \mathrm{~Hz}$ and an extra repetition rate of $12.5 \mathrm{~Hz}$. The parameters of the signals investigated fall in these ranges.

In addition, Polish directives recommend that the duration of the intermittent signals should be equal to the duration of the pause between succeeding signals. The acoustic signalling devices installed at pedestrian crosswalks in Poznań emit signals of duration much shorter than the pause between succeeding signals and their time pattern cannot be changed. It was the reason why two additional types of signals which fulfil this condition were studied.

Therefore three types of intermittent sounds of different time pattern shape (rectangular (RR, RS) and triangular (TS)) filled up with different waves (rectangular (RR) and sinusoidal (RS, TS)) were selected as potential candidates for the best signal. The signals with rectangular time pattern filled up with rectangular wave (RR) are presently generated by the acoustic signalling devices at pedestrian crosswalks in Poznań. The duration of the RR signal was much shorter than the pause between succeeding signals. Two other signals, the one with rectangular time pattern filled up with sinusoidal wave (RS) and the one with triangular time pattern filled up with sinusoidal wave (TS) were proposed by us. In these signals the duration of the signal and pause between the subsequent signals were of the same length.

\section{Method}

Three experiments were conducted. Experiment I was concerned with the calibration. The aim of experiment I was to verify whether the hearing thresholds known from literature for tones of a given frequency are the same for the investigated intermittent signals with different shapes of time pattern. Result of experiment I should indicate which signals have the lowest hearing threshold. The aim of experiment II was to find which signal is detected at the lowest signal to noise ratio (SNR) when presented against the background of four types of environmental noise. Results of experiment III should identify the least annoying signals. It should be mentioned that the results of annoyance assessment was considered as a less important factor comparing to value of SNR measured in the background of environmental noises (experiment II).

\subsection{Stimuli}

Three types of signals were studied (Fig. 1). The first signal was an intermittent signal with a rectangular time pattern filled up with rectangular wave (RR) generated by the acoustic signalling devices which are popular installations at pedestrian crosswalks in Poznań (Fig. 1, top part). The second signal was an intermittent signal with a rectangular time pattern filled up with sinusoidal wave (RS, Fig. 1, central part) and the third signal was an intermittent signal with a triangular time pattern filled up with sinusoidal wave (TS, Fig. 1, bottom part). Both the RS and TS signals were generated in Matlab environment.
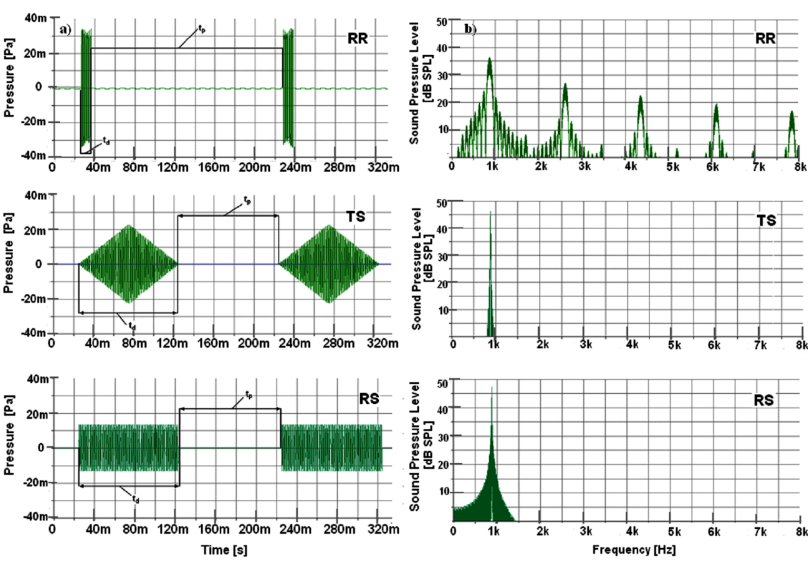

Fig. 1. Examples of signals with fundamental frequency of $880 \mathrm{~Hz}$ and repetition rate of $5 \mathrm{~Hz}\left(t_{\mathrm{d}}-\mathrm{du}-\right.$ ration of signal, $t_{\mathrm{p}}$ - duration of pause). Time pattern (a) and the average power spectra (b) of intermittent signals with rectangular time pattern filled up rectangular wave (RR - top part), intermittent signals with triangular time pattern filled up sinusoidal wave (TS central part) and intermittent signals with a rectangular time pattern filled up sinusoidal wave (RS - bottom part).

Parameters of the signals studied were consistent with the current normative regulations and partly determined by the acoustics parameters of the signalling devices which were already installed at pedestrian crosswalks in Poznań. The choice of the repetition rate of the studied signals $(5 \mathrm{~Hz}$ and $9 \mathrm{~Hz}$ ) was due to the results of a pilot study [10]. In this study people were asked to judge annoyance of signals with different repetition rate on a three point annoyance scale: "not annoying", "don't know", "annoying". The results show that above $50 \%$ of respondents assessed signals with repetition rate above $9 \mathrm{~Hz}$ as "annoying".

For the sequence of RR signals the duration of the signal was much shorter than the pause between succeeding signals. For the signals with a repetition rate of $5 \mathrm{~Hz}$ the duration of the signal was $10 \mathrm{~ms}$ and the duration of the pause was $190 \mathrm{~ms}$. For the signals with a repetition rate of $9 \mathrm{~Hz}$ the signal duration was $5 \mathrm{~ms}$ and that of the pause $105 \mathrm{~ms}$. In the sequence of RS and TS signals the duration of the signals was equal to the duration of the pause between succeeding signals (according to Polish directives). For the $\mathrm{RS}$ and TS signals with a repetition rate of $5 \mathrm{~Hz}$ the duration of the signal and pause was $100 \mathrm{~ms}$. For these signals with a repetition rate of $9 \mathrm{~Hz}$ the duration of the signal and the pause was $55 \mathrm{~ms}$.

The fundamental frequencies were chosen to be the lowest, the highest and two middle frequencies from the range recommend by current Polish directives. The sig- 
nals studied had the following fundamental frequencies $\left(F_{0}\right): 550 \mathrm{~Hz}, 880 \mathrm{~Hz}, 1580 \mathrm{~Hz}$ and $2000 \mathrm{~Hz}$, and the following repetition rate $\left(f_{\mathrm{r}}\right): 5 \mathrm{~Hz}$ and $9 \mathrm{~Hz}$. The fundamental frequency of $880 \mathrm{~Hz}$ is recommended for tone signals by Polish Norm PN-Z-80100-2004 and literature as the best in the aspect of detection against a background noise [11] and regarding the absorption by windows' panels, so it is not annoying [12]. The selected repetition rates were $5 \mathrm{~Hz}$ and $9 \mathrm{~Hz}$ - the lowest recommended by current Polish regulations and the highest repetition rate at which signal is not annoying.

Four types of environmental noise usually occurring at pedestrian crosswalks, generated by cars, trams, buses and trucks were used as a background noise.

\subsection{Participants}

The persons (18-28 years of age) with normal vision and normal hearing (hearing thresholds did not exceed $25 \mathrm{~dB}$ HL for any frequency) were selected for the study. Ten persons took part in experiment I and experiment II and eight persons took part in experiment III. We selected persons with normal vision because there are no differences in auditory sensitivity between persons with and without vision impairments [13-15].

\subsubsection{Experiment I}

\subsection{Procedure and equipment}

The sound pressure level of the signals tested was adjusted in the programs: PULSE B\&K version 12.6 and Wave Lab version 4.0. Figure 2 shows a block diagram of the system used in this study. The stimuli recorded on a CD disk were played back via a CD player to an audiometer Madsen OB 822. The stimuli were presented monaurally by the circumaural earphone Sennheiser HAD 200 connected to the audiometer. The system was calibrated using an artificial ear (B\&K type 4152) and a sonometer (B\&K type 2204).

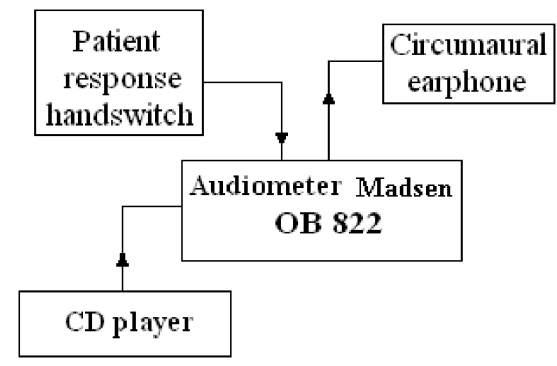

Fig. 2. A block diagram of the system used in the study.

The hearing thresholds were measured for 24 signals (8 representing each type of signals: RR, RS, TS) by using the method of limits, with steps of $1 \mathrm{~dB}$. In experiment I the subjects were instructed to push the patient response handswitch when they perceived a signal. The procedure was repeated as long as the subject responded three times at the same level. The signals were presented in a random order. Experiment I consisted of three listening sessions and lasted for about one hour. A single listening session lasted for about $15 \mathrm{~min}$ and its length depended on the repeatability of the subjects' response. Each listening sessions was followed by a five-minute break.

\subsubsection{Experiment II}

The sound pressure level of signals tested was adjusted in the programs: PULSE B\&K version 12.6 and Wave Lab version 4.0. The noises were specially corrected in the program Wave Lab version 4.0 in order to get a level of noise (stationary signal) constant in time with an average spectrum the same as that of a single pass-by of a given category of vehicle. The corrected traffic noises had sound levels equal to $70 \mathrm{~dB}(\mathrm{~A})$. The value of the sound level of the traffic noise was taken from the Acoustic Map of Poznań [16].

Two-channel recordings were prepared, with the traffic signals recorded in one channel and the traffic noises in the other channel. The measuring system was the same as in experiment I (Fig. 2). The stimuli were recorded on a CD disk and delivered via a CD player to a two-channel audiometer Madsen OB 822. The stimuli were presented monaurally (noise and signal to the same ear) by the circumaural earphone Sennheiser HAD 200 connected to the audiometer. The system was calibrated using an artificial ear (B\&K type 4152) and a sonometer (B\&K type 2204).

The detection thresholds of the signals studied in background noise were measured for 24 signals and 4 types of noise (32 for each type of signals: RR, RS, TS) using the method of limits, with steps of $1 \mathrm{~dB}$. The instruction for subjects and measurements' procedure in experiment II was similar to that in experiment I. The difference was that in experiment II the signals were detected in the background of environmental noise. The signals were presented in a random order.

After fixing the level of noise to $70 \mathrm{~dB}$ (A) and delivering it to the earphone, the detection thresholds of the signals studied in noise were evaluated. In the experiment the SNR was found according to formula (1):

$$
\mathrm{SNR}=L_{\mathrm{s}}-L_{\mathrm{n}}[\mathrm{dB} \mathrm{A}],
$$

where $L_{\mathrm{S}}$ is the minimum level of a signal which was perceived in the background noise, $L_{\mathrm{n}}$ is the level of background noise (the same for all types of traffic noise: $70 \mathrm{~dB}(\mathrm{~A}))$.

Experiment II consisted of six listening sessions and lasted for about two and a half hours. A single listening session took about 15-20 min. Each listening sessions was followed by a five-minute break. Three listening sessions were followed by a fifteen-minute break.

\subsubsection{Experiment III}

The sound pressure levels of the signals tested were adjusted in the programs: PULSE B\&K version 12.6 and Wave Lab version 4.0. The stimuli were presented binaurally by circumaural earphone Sennheiser HD 600 in a sound-proof chamber. The stimuli were recorded on a 
CD disk and played back via an equalizer PEQ IV and an earphone distributor B\&K to the earphones. The earphone distributor made it possible to connect eight sets of earphones so that eight participants could take part in the experiment simultaneously.

24 signals were presented to the participants in experiment III. Each signal was repeated three times. To assess the signal annoyance the participants were asked to respond by marking a number on an 11-point numerical scale on the response sheet, according to ICBEN scale (Table).

\section{TABLE}

The ICBEN numerical and verbal scales of annoyance of signals. The subjects were asked to write the number describing annoyance assessment of the signals in a frame box.

\begin{tabular}{ccccccccccr}
\hline \hline \multicolumn{10}{c}{ Signal is annoying } \\
not at all & a little & rather & \multicolumn{1}{c}{ substantially } & extremely \\
\hline 0 & 1 & 2 & 3 & 4 & 5 & 6 & 7 & 8 & 9 & 10
\end{tabular}

According to ICBEN (International Commission on the Biological Effects of Noise), annoyance is evaluated in a 5-point verbal scale and a corresponding $0-10$ point numerical scale. The same 5-point verbal annoyance scale (not at all, a little, rather, substantially, extremely annoying) is used in ten countries (in nine different languages) $[17,18]$. Experiment III lasted for about thirty five minutes. Each signals duration was $10 \mathrm{~s}$ with $2 \mathrm{~s}$ response time.

\section{Results}

\subsection{Experiment I}

The analysis of variance (ANOVA) showed that the effect of the fundamental frequency of the signal was statistically significant $(F(3,216)=25.0 ; p<0.001)$. The average hearing thresholds for the signals of a fundamental frequency $550 \mathrm{~Hz}$ were about $11 \mathrm{~dB}$ SPL, for those of a fundamental frequency $880 \mathrm{~Hz}$ the threshold was from $7 \mathrm{~dB}$ SPL to $9 \mathrm{~dB}$ SPL, for those of a fundamental frequency $1580 \mathrm{~Hz}$ it varied from $4 \mathrm{~dB}$ SPL to $6 \mathrm{~dB}$ SPL and for those of $2000 \mathrm{~Hz}$ it varied from $6 \mathrm{~dB}$ SPL to $8 \mathrm{~dB}$ SPL (Fig. 3, left part).

No effect was detected of the type of signal $(F(2,216)=1.52 ; p>0.2)$ and the repetition rate $(F(1,216)=7.45 ; p>0.05)$. No statistically significant differences in perception of different types of signals (RR, RS and TS) of the same parameters $\left(F_{0}\right.$ and $\left.f_{\mathrm{r}}\right)$ were observed. Signals with the repetition rate of $5 \mathrm{~Hz}$ were perceived easier than those with the repetition rate of $9 \mathrm{~Hz}$ but the effect was not statistically significant (Fig. 3, right part).

Although short, intermittent sounds were studied, the results of experiment I were compared to the reference equivalent threshold sound pressure levels for a constant

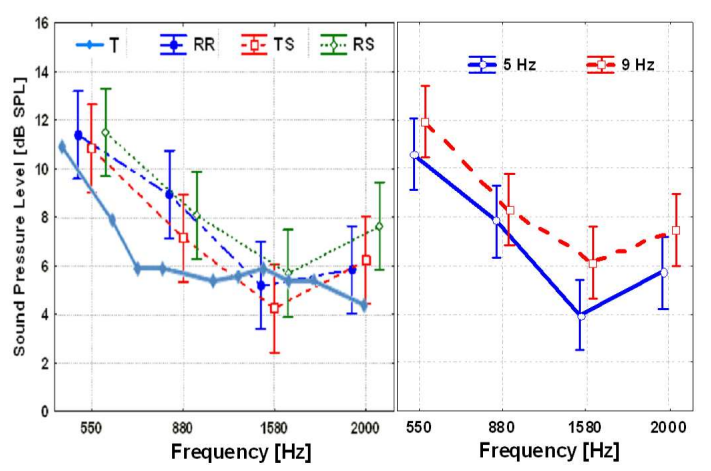

Fig. 3. The average hearing thresholds of RR, TS and RS signals (broken lines) in relation to reference equivalent threshold sound pressure level for circumaural earphones - according to EN ISO 389-8:2004, for earphone SENNHEISER HDA 200 ( T - solid line, left part) and the effect of repetition rate on the average hearing thresholds (right part).

test-tone, for circumaural earphone which were used in the study (EN ISO 3898:2004). Figure 3 (left part) shows that the average value of hearing thresholds of signals having definite fundamental frequencies: $550 \mathrm{~Hz}$, $880 \mathrm{~Hz}, 1580 \mathrm{~Hz}$ and $200 \mathrm{~Hz}$ conforms to the accuracy of $3 \mathrm{~dB}$ with the values of the reference equivalent threshold sound pressure levels for circumaural earphone. The largest difference was noticed for the signals with the fundamental frequency $880 \mathrm{~Hz}$ and $2000 \mathrm{~Hz}$. These signals had higher hearing thresholds than those of a constant test-tone known from literature.

\subsection{Experiment II}

The ANOVA showed that the type of noise, type of signal and fundamental frequency affected the results of the experiment (Fig. 4).

The effect of the type of noise was statistically significant $(F(3,960)=62.9 ; p<0.001)$, (Fig. 4, top part). The SNR ratios for the signals perceived in the background of tram noise were the smallest and were in a range from $-32 \mathrm{~dB}$ to $-18 \mathrm{~dB}$ for $880 \mathrm{~Hz}$, for car, bus and truck noise SNR ratios were in a range from $-27 \mathrm{~dB}$ to $-10 \mathrm{~dB}$ for $880 \mathrm{~Hz}$.

The effects of type of signal $(F(2,960)=71.41 ; p<$ $0.001)$ and fundamental frequency $(F(3,960)=114.0$; $p<0.001$ ) were also statistically significant (Fig. 4 , top part). The same tendency in signal perception (depending on fundamental frequency) in the background of traffic noise was observed for RS and TS signals. For these signals no statistically significant differences were observed. A completely different tendency was observed for RR signals. The largest differences between the SNR ratio values were observed for the signals of the fundamental frequency $880 \mathrm{~Hz}$ - from $10 \mathrm{~dB}$ for tram noise to $15 \mathrm{~dB}$ for the remaining types of noise. For this fundamental frequency the SNR ratio for RS and TS signals reached the value from $-18 \mathrm{~dB}$ to $-10 \mathrm{~dB}$, for RR signals 


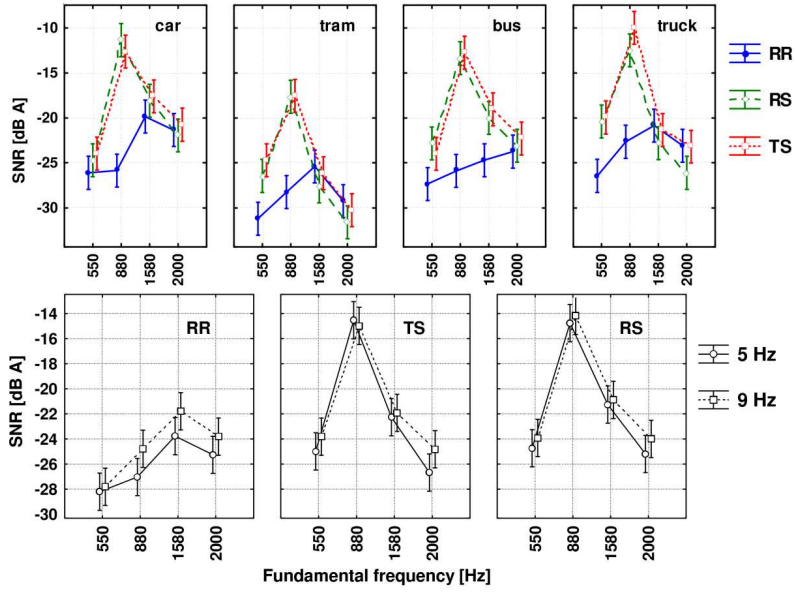

Fig. 4. The effect of the type of noise, type of signal and fundamental frequencies (top part) and the effect of the repetition rate, type of signal and fundamental frequency (bottom part) on the detection thresholds in a background traffic noise (SNR ratio values).

the SNR ratio was from $-28 \mathrm{~dB}$ to $-23 \mathrm{~dB}$. Much smaller differences were observed between the SNR ratio for RS, TS and RR signals of the fundamental frequency $550 \mathrm{~Hz}$ (about $5 \mathrm{~dB}$ for tram, bus and truck noise, no statistically significant differences for car noise were observed). For the fundamental frequencies $1580 \mathrm{~Hz}$ and $2000 \mathrm{~Hz}$, no statistically significant differences between the SNR ratio values for RR, RS and TS signals were observed (except for the signal of the fundamental frequency $1580 \mathrm{~Hz}$ perceived in the background of bus noise).

No statistically significant differences in perception of $\mathrm{RR}, \mathrm{RS}$ and TS signals with repetition rate of $5 \mathrm{~Hz}$ and $9 \mathrm{~Hz}$ in the background of noise were observed (Fig. 4, bottom part).

\subsection{Experiment III}

The ANOVA showed that all factors tested in experiment I influenced the results of annoyance assessment.
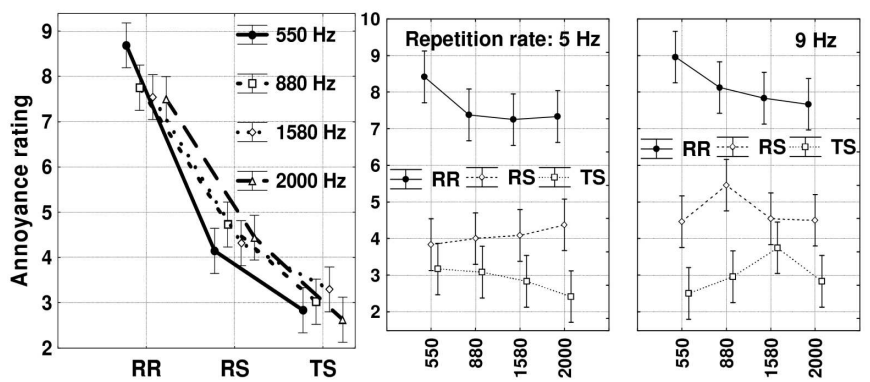

Fig. 5. The effect of the type of signal and fundamental frequency (left part) and fundamental frequency and repetition rate (right part) on annoyance assessment.

The effect of the type of signal was statistically significant $(F(2,384)=1166.5 ; p<0.001)$. RR signals were evaluated as very annoying (assessment about 8 - "substantially"), RS signals as annoying (assessment about 4, 5- "rather") whereas TS signals as the least annoying (assessment about 3- near "a little"), (Fig. 5, left part). The fundamental frequency was also a statistically significant factor $(F(3,384)=3.62 ; p<0.002)$. The tendency in annoyance assessment of the signals with various fundamental frequencies was not observed (Fig. 5, right part).

The effect of repetition rate was statistically significant $F(1,384)=27.84 ; p<0.001$. The signals with the repetition rate of $5 \mathrm{~Hz}$ were perceived as less annoying than those with the repetition rate of $9 \mathrm{~Hz}$.

\section{Discussion}

\subsection{Experiment I}

The hearing thresholds known from literature could approximate those for RR, RS and TS signals measured via circumaural earphone. We showed that different time patterns of the above signals did not affect the value of hearing threshold in the quiet. From the point of view of hearing loss which is related to the age it is not recommended to use signals of the fundamental frequency of $2000 \mathrm{~Hz}$. On the basis of the results of experiment I it is not recommended to use signal of fundamental frequencies of $500 \mathrm{~Hz}$ and mentioned before signal of fundamental frequency of $2000 \mathrm{~Hz}$.

The best signals when taking into account the hearing thresholds in quiet are any type of intermittent sounds (RR, RS and TS) of the fundamental frequencies $880 \mathrm{~Hz}$ and $1580 \mathrm{~Hz}$, regardless of the repetition rate.

\subsection{Experiment $I I$}

Analysis of the results of experiment II shows the differences in SNR values between the RR and sinusoidal signals (RS, TS). The largest differences occur for the signal of the fundamental frequency of $880 \mathrm{~Hz}$. One of possible explanations of this effect is the presence of maxima in the spectra of these noises in the region of this frequency (Fig. 6).

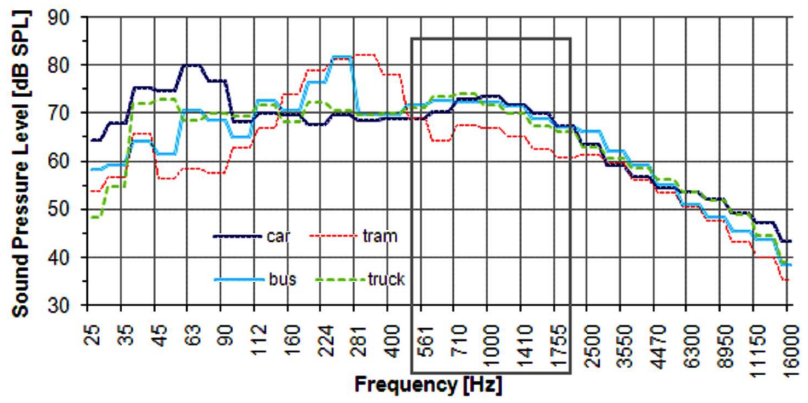

Fig. 6. Spectra of noises generated by cars, trams, buses and trucks; the range of fundamental frequencies of the signals studied are marked. 
However, the question is why their presence does not influence the RR signal. As can be seen in Fig. 1 the RR signal has higher harmonics which are absent in sinusoidal signals. The presence of these extra harmonics makes the RR signal less sensitive to the maxima of environmental noises. Therefore for this fundamental frequency, i.e. for $880 \mathrm{~Hz}$, the sinusoidal signals, i.e. intermittent sounds with rectangular time pattern filled up with sinusoidal wave (RS) and with triangular time pattern filled up with sinusoidal wave (TS) are not recommended.

All types of signals (RR, RS, TS) of the fundamental frequency of $2000 \mathrm{~Hz}$ are perceived in a similar way in the background of environmental noise. As it was mentioned because of a hearing loss which is related to the age, it is not recommended to use signals of the fundamental frequency of $2000 \mathrm{~Hz}$. Therefore, the best type of signals from the point of view of signal detection in the background of environmental noises are RR ones of the fundamental frequencies of $550 \mathrm{~Hz}, 880 \mathrm{~Hz}$ or $1580 \mathrm{~Hz}$ and $\mathrm{RS}$ or TS signals of the fundamental frequency of $1580 \mathrm{~Hz}$, regardless of the repetition rate.

On the other hand, the detection of a signal in the background of noise depends on the type of noise. The SNR values for the tram noise were the lowest. This result confirms the existence of the "tram bonus" [19]. However, when choosing the best signal for pedestrian crosswalk we should concentrate on the worst conditions, i.e. the highest values of SNR. The highest SNR values were obtained for car and truck environmental noises. Finally, we can recommend, besides the signal of $880 \mathrm{~Hz}$ also those of the fundamental frequency of $1580 \mathrm{~Hz}$. It is worth noting that for this frequency $(1580 \mathrm{~Hz})$ there are no significant differences between the SNR values for all types of signals and almost all environmental noises.

\subsection{Experiment III}

If the criterion of the signal choice was its annoyance we should use RS or TS signals with the repetition rate of $5 \mathrm{~Hz}$ at pedestrian crosswalks (TS - a little annoying, RS - rather annoying). However, annoyance was considered as a secondary factor in relation to the value of SNR measured in the background of traffic noise. For a given sound level, annoyance should not be a factor determining the choice of a signal. The TS and RS signals have similar average loudness and sharpness but the RR signals have a higher loudness index $N(5)$ (about 7 sone) and sharpness index $N(5)(3-4$ acum) than TS and RS signals (loudness index $N(5)$ about 3-4 sone, sharpness index $N(5)$ about 0.5-2 acum). For this reason the most annoying were RR signals (substantially annoying).

The signals with the repetition rate of $5 \mathrm{~Hz}$ were evaluated as less annoying than those with the repetition rate of $9 \mathrm{~Hz}$. It is known that the loudness grows with increasing repetition rate of signals and leads to higher annoyance ratings of these signals [20].

\section{Conclusion}

The above presented and discussed results permit drawing the following conclusions.

1. On the basis of the results of experiment I and experiment II the best signals are those with a rectangular time pattern filled up with rectangular wave (RR) of the fundamental frequencies $880 \mathrm{~Hz}$ or $1580 \mathrm{~Hz}$ and the signals with a rectangular time pattern (RS) or with triangular time pattern (TS) both filled up with sinusoidal wave of the fundamental frequencies $1580 \mathrm{~Hz}$, regardless of the repetition rate.

2. On the basis of the results of experiment III the best signals are those with a triangular time pattern filled up with sinusoidal wave (TS) and those with a rectangular time pattern filled up with sinusoidal wave (RS) of the fundamental frequencies $880 \mathrm{~Hz}$ and $1580 \mathrm{~Hz}$ and both with repetition rate of $5 \mathrm{~Hz}$.

3. The signals satisfying both criterions are those with a triangular time pattern (TS) and those with a rectangular time pattern (RS) both filled up with sinusoidal wave of the fundamental frequencies $1580 \mathrm{~Hz}$ and repetition rate of $5 \mathrm{~Hz}$.

4. Because the annoyance assessment was considered as a secondary factor in relation to the SNR value measured in the background of environmental noises, we recommend the use of one of the four signals $\left(F_{0}, f_{\mathrm{r}}\right)$ : RR $(880 \mathrm{~Hz}, 5 \mathrm{~Hz}), \mathrm{RR}(1580 \mathrm{~Hz}$, $5 \mathrm{~Hz})$, TS $(1580 \mathrm{~Hz}, 5 \mathrm{~Hz})$ or RS $(1580 \mathrm{~Hz}, 5 \mathrm{~Hz})$.

\section{Acknowledgments}

The work was supported by the National Centre for Investigation and Development, grant N R11 0008 04, April 2008, Warsaw, Poland.

\section{References}

[1] A.Y.J. Szeto, N.C. Valerio, R.Ł. Novak, J. Rehabil. Res. Dev. 28, 57 (1991).

[2] A.C. Scott, J.M. Barlow, B.L. Bentzen, T.L.Y. Bond, D. Gubbe, J. Transportation Research Board 2073, 94 (2008).

[3] B.L Bentzen, J.M. Barlow, L. Franck, Institute of Transportation Engineers J. 70, 32 (2000).

[4] A.Y.J. Szeto, N.C. Valerio, R.Ł. Novak, J. Rehabil. Res. Dev. 28, 71 (1991).

[5] S.R. Wall, D.H. Ashmead, B.L. Bentzen, J. Barlow, Ergonomics 47, 1318 (2004).

[6] G.R. Watts, N.S. Godfrey, T.A. Savill, Appl. Acoust. 62, 15 (2001).

[7] List of Act No. 220-.2003, entry 2181 of 23.12 (annex no. 3 point 3.3.5.), Decree of Ministry of Infrastructure, Detailed engineering conditions for road signals and conditions place them on the roads (2003). 
[8] Polish Norm PN-Z-80100-2004, Technical devices for blind persons and persons who have low vision. Sound signalization at the pedestrian crossing with light signalization, 2004 (in Polish).

[9] ISO 23600-2007(E), Assistive products for persons with vision impairment and persons with vision and hearing impairments - Acoustic and tactile signals for pedestrian traffic lights, 2007 (in Polish).

[10] E. Bogusz, M.A. Thesis, Adam Mickiewicz University, Poznań 2008.

[11] J.M. Barlow, B.L. Bentzen, L.S. Tabor, Accessible pedestrian signals: synthesis and guide to best practice, NCHRP 3-62, Final report, Web-Only Document, 2003.

[12] T. Poulsen, Appl. Acoust. 15, 363 (1982).

[13] K. Buerklen, in: Tyflopedagogy, Ed. Z. Sękowska, WSiP, Warsaw 1981, p. 72, Ch. I (in Polish).

[14] I. Starlinger, W. Niemeyer, Int. J. Audiol. 20, 503 (1981).
[15] M. Bross, M. Borenstein, Percept. Mot. Skills 55, 963 (1982).

[16] M. Duś, W. Babicz, Measurements of noise in the environment of national and of a province roads in area of Poznań city, Adam Mickiewicz University, Poznań 2006 (in Polish).

[17] A. Preis, T. Kaczmarek, H. Wojciechowska, J. Żera, J.M. Fields, Int. J. Occup. Med. Environ. Health 16, 155 (2003).

[18] J.M. Fields, R.G. De Jong, T. Gjestland, I.H. Flindell, R.F.S. Job, S. Kurra, P. Lercher, M. Vallet, T. Yano, J. Sound Vib. 242, 641 (2001).

[19] T. Kaczmarek, H. Hafke, A. Preis, S. Sandrock, B. Griefahn, T. Gjestland, Arch. Acoust. 31, 405 (2006).

[20] E. Zwicker, H. Fastl, Psychoacoustics Facts and Models, Springer Verlag, Berlin 1999. 